\title{
THE EFFECT OF INCIDENT LIGHT POLARIZATION ON VEGETATION BIDIRECTIONAL REFLECTANCE FACTOR
}

\author{
G.T. Georgiev ${ }^{a}$, K. Thome ${ }^{b}$, K.J. Ranson ${ }^{b}$, M. King ${ }^{c}$, J.J. Butler ${ }^{b}$

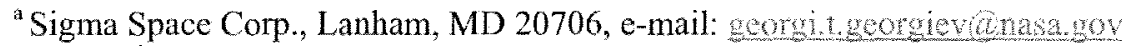 \\ ${ }^{b}$ NASA Goddard Space Flight Center, Greenbelt, MD 20771 \\ 'University of Colorado, Boulder, CO 80309
}

The laboratory-based Bidirectional Reflectance Factor (BRF) polarization study of vegetation is presented in this paper. The BRF was measured using a short-arc Xenon lamp/monochromator assembly producing an incoherent, tunable light source with a welldefined spectral bandpass at visible and near-infrared wavelengths of interest at $470 \mathrm{~nm}$ and $870 \mathrm{~nm}$ and coherent light source at 1.656 microns. All vegetation samples were measured using $\mathrm{P}$ and $\mathrm{S}$ linearly polarized incident light over a range of incident and scatter angles. By comparing these results, we quantitatively examine how the BRF of the samples depends on the polarization of the incident light. The differences are significant, depend strongly on the incident and scatter angles, and can be as high as $120 \%$ at 67 deg incident and $470 \mathrm{~nm}$.

The global nature of Earth's processes requires consistent long-term calibration of all instruments involved in data retrieval ${ }^{l}$. The BRF defines the reflection characteristics of Earth surface. It provides the reflectance of a target in a specific direction as a function of illumination and viewing geometry. The BRF is a function of wavelength and reflects the structural and optical properties of the surface. Various space and airborne radiometric and imaging remote sensing instruments are used in the remote sensing characterization of vegetation canopies ${ }^{2}$ and soils ${ }^{3}$, oceans ${ }^{4}$, or especially large pollution sources ${ }^{5}$. The satellite data is validated through comparison with airborne, ground-based and laboratory-based data in an effort to fully understand the vegetation canopy reflectance. The Sun's light is assumed to be unpolarized at the top of the atmosphere; however it becomes polarized to some degree due to atmospheric effects by the time it reaches the vegetation canopy. Although there are numerous atmospheric correction models, laboratory data is needed for model verification and improvement.

The BRF data of this study was obtained using the scatterometer located in NASA's Goddard Space Flight Center Diffuser Calibration Laboratory (DCL). The DCL scatterometer can perform in-plane and out-of-plane BRF measurements with typical measurement 
uncertainties of $1 \%(k=1)$. The accuracy of measured BRF depended on the signal-to-noise ratio and was determined by a sample's spatial optical scatter properties. The same sample's spectral reflectance was also measured with an Analytical Spectral Devices (ASD) spectroradiometer at the same geometries in the spectral range from 350 to $2500 \mathrm{~nm}$ in order to more completely characterize the sample's spectral reflectance properties.

The diffuser samples calibrated in our Facility exhibit different BRF depending on the polarization of the incident light ${ }^{6}$. Now we expand our long-term calibration study of diffuser standards to vegetation samples with the goal to better understand the remote sensing data of air- and space-based instruments. There are a number of well respected studies on polarized reflectance from leaves producing a number of publications ${ }^{2,7}$, however little attention has been devoted to the case of polarized source and unpolarized detector which is pretty common instrument architecture especially using lidar techniques.

This paper presents BRF data of lab studied vegetation samples, namely, fresh and dry leaves from the tulip poplar tree (Liriodendron tulipifera), and black locust tree (Robinia pseudoacacia) located in Maryland, U.S. ${ }^{8}$ The vegetation samples were measured in-plane and out-of-plane at $0^{\circ}$ and $67^{\circ}$ incident angles, from $0^{\circ}$ to $80^{\circ}$ scatter zenith angles and from $0^{\circ}$ to $180^{\circ}$ scatter azimuth angles in steps of $5^{\circ}$. The BRF at $\mathrm{P}$ and $\mathrm{S}$ linearly polarized incident light was evaluated relative to the BRF of unpolarized incident light. The difference in BRF dependence on the polarization state of the incident light and varies with the measurements angular setup. For example, the BRF dependence on incident light polarization at $0^{\circ}$ incident angle increases with increasing scatter zenith angles and decreases with increasing wavelength at fixed scatter azimuth angle. Also the BRF difference in using $\mathrm{S}$ and $\mathrm{P}$ polarized incident light is larger at $90^{\circ}$ scatter azimuth angle and exhibits a minimum at $45^{\circ}$ and $135^{\circ}$ scatter azimuth angles. The in-plane BRF dependence on $\mathrm{S}$ and $\mathrm{P}$ polarized incident light at $67^{\circ}$ incident angle has a maximum at the specular direction and again is lower at higher wavelengths and lower scatter zenith angles. However, the out-of-plane BRF difference is more complex. It is higher at $90^{\circ}$ scatter azimuth and lower at $45^{\circ}$ and $135^{\circ}$ scatter azimuth.

The differences in BRF at $\mathrm{P}$ and $\mathrm{S}$ polarized incident light can be traced back to the vegetation reflectance spectra and is based on vegetation biochemical composition. The shorter the wavelength the higher the absorption of $\mathrm{S}$ or $\mathrm{P}$ polarized incident light due to the absorption 
of the light into the leaf structure as the leaf pigments absorb mainly before the red edge. Leaves usually reflect weakly in the blue and red wavelengths because of absorption by pigments, and strongly in the near-infrared due to cellular refraction.

The BRF dependence on the polarization of the incident light is best described with the electronic transition of pigments to the excited singlet state, whether the dipoles are parallel to the oscillating electric vector of the incident polarized light. The light will be then preferentially absorbed thus reducing the reflection from the specimen. New data is presented describing the dependence on the scatter azimuth and zenith angles of incident light. The polarization effect on BRF is pronounced the best at in-plane and out-of-plane geometries that coincide with $\mathrm{P}$ and/or $\mathrm{S}$ polarization of the incident light. In turn it is smaller when the scatter azimuth angle is $45^{\circ}$ from the $\mathrm{P}$ and/or $\mathrm{S}$ polarization of the incident light.

The laboratory results indicate that the often overlooked impact of polarization effects in the reflectance of vegetation may need to be considered as the requirements on accuracy of remote sensing data continue to become more stringent. The results from studies such as the one presented here will be of interest to the remote sensing community both in developing sensor design requirements and in providing constraints for modeling and correction efforts of airborne and satellite-based data.

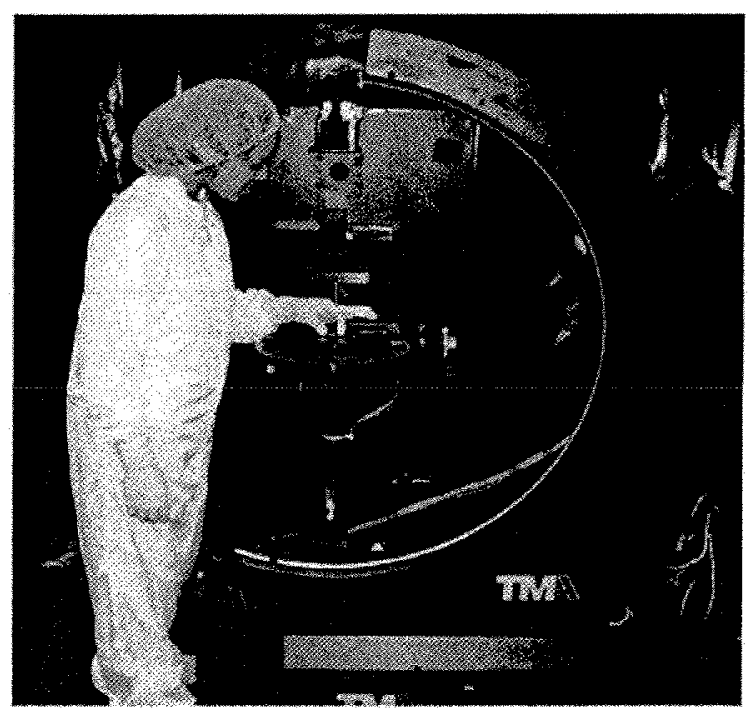

Fig. 1: The Scatterometer 


\section{BIBLIOGRAPHY}

${ }^{1}$ J.J. Butler, B.C. Johnson, R.A. Barnes, "The calibration and characterization of Earth remote sensing and environmental monitoring instruments", in Optical Radiometry, Ed. A.C. Parr, R.U. Datla, J.L. Gardner, Academic Press, New York (2005),

${ }^{2}$ K.J. Ranson, J.R. Irons, D.L. Williams, "Multispectral bidirectional reflectance of Northern forest canopies with the Advanced Solid-State Array Spectroradiometer (ASAS)", Remote Sens. Environ., 47, 2, 276-289 (1994)

${ }^{3}$ C.K. Gatebe, M.D. King, S. Platnick, G.T. Arnold, E.F. Vermote, B. Schmid, "Airborne spectral measurements of surface-atmosphere anisotropy for several surfaces and ecosystems over southern Africa", J. Geophysical Research, 108, D13, 25-1-16 (2003).

${ }^{4}$ C.K. Gatebe, M.D. King, A.I. Lyapustin, G.T. Arnold, J. Redemann, "Airborne spectral measurements of ocean directional reflectance", J. Atmospheric Sciences, 62, 1072-1092 (2005).

${ }_{5}^{5}$ M.D. King, "Directional and spectral reflectance of the Kuwait oil-fire smoke", J. Geophysical Research, 97, D13, 14545-14549 (1992).

${ }^{6}$ G.T. Georgiev, J.J. Butler: "The effect of incident light polarization on Spectralon BRDF measurements", Proc. SPIE Sensors, Systems, and Next-Generation Satellites VIII., 5570, 492-502, (2004)

${ }^{7}$ D.P. Gibbs, C.L. Betty, A.K. Fung, A.J. Blanchard, J.R. Irons: "Automated measurement of polarized bidirectional reflectance", Remote Sens. Environ., 43, 97-114 (1993)

${ }^{8}$ G.T. Georgiev, C.K. Gatebe, J.J. Butler, M.D. King: "Laboratory and airborne bidirectional reflectance distribution function analysis of vegetation leaves and soil samples", Transactions of IEEE Geoscience and Remote Sensing, 47, 8, 2546-2556 (2009) 